\title{
EL EMPRENDIMIENTO EN PERSONAS CON DISCAPACIDAD. UNA APROXIMACIÓN AL CONTEXTO SOCIOLABORAL
}

\author{
Autores: Salvador Manzanera-Román \\ smanzanera@um.es \\ Universidad de Murcia \\ Ángel Olaz Capitán \\ olazcapi@um.es \\ Universidad de Murcia \\ Pilar Ortiz García \\ portizg@um.es \\ Universidad de Murcia
}

\section{Resumen \\ Este artículo se enmarca dentro del proyecto Discapacidad y emprendimiento. Análisis competencial (CSO2016-75818-R). El objetivo principal es conocer el pa- pel que desempeñan los conocimientos, capacidades y habilidades de las personas con discapacidad orgánica, física y sensorial a la hora de explicar sus acciones de emprendimiento. En la fase inicial se fijaron varios objetivos específicos como la caracterización de la población con discapacidad, el conocimiento de su posición}


en el mercado laboral, y el análisis del papel de las políticas activas de empleo para superar las barreras al emprendimiento del colectivo objeto de estudio.

La hipótesis de la investigación considera que las personas con discapacidad están en una posición desfavorable para conseguir un empleo y desarrollar una actividad emprendedora.

Metodológicamente, la investigación tiene un doble enfoque, cuantitativo y cualitativo, aunque en la fase inicial se limitó a una revisión documental junto a un análisis estadístico de fuentes secundarias de información sobre el tema para el período 2008-2016 en España: El empleo de las personas con discapacidad, el Informe Anual del Mercado de Trabajo de las Personas con Discapacidad y la Encuesta de Condiciones de Vida.

El análisis de los resultados determina que las personas con discapacidad cuentan con importantes dificultades para acceder al mercado de trabajo y al emprendimiento, por lo que se impone un ejercicio de sensibilización y visibilización de estos colectivos ante la sociedad con medidas y acciones concretas que destaquen sus aportaciones y favorezcan su inclusión sociolaboral.

Palabras clave: emprendimiento; discapacidad; mercado de trabajo; políticas de empleo.

\section{Entrepreneurship of disabled people. An approach to social and labour context}

\section{Abstract}

This paper entitled "Entrepreneurship of disabled people. Socio-labour contextualization" is part of the Disability and entrepreneurship project. Competencial analysis (CSO2016-75818-R), funded by the MINECO, whose main goal is to analyze the role played by competences -understood as a set of knowledge, skills and abilities- in people with physical and / or sensory disabilities, in the design, development and performance in the field of entrepreneurship. In the initial phase, several specific goals were determined: firstly, showing some distinctive features about the social and labor situation of people with disabilities; secondly, analyzing the role of the active labour policies to overcome the barriers to entrepreneurship of disabled people.

The hypothesis of the research considers that people with disabilities are in an unfavourable position to get a job and develop an entrepreneurial activity.

Methodologically, the research has twofold approach: quantitative and qualitative techniques. However, in the initial phase is limited to a documentary review and statistical analysis with secondary sources on the subject for the period 
2008-2016 in Spain: The Employment of Disabled People, The Annual Report of the Labour Market of Disabled People and Living Conditions Survey.

The analysis of the results determines that disabled people have significant difficulties to access to the labour market and entrepreneurship activity. Therefore, it is compulsory to make disabled people visible and to make the people aware of this collective by means of actions that highlight their contributions and foster their socio-labour inclusion.

Key words: entrepreneurship; disability; labour market; labour policies.

Fecha de recepción: 01/02/2019

Fecha de aceptación: 09/04/2019

\section{PLANTEAMIENTO GENERAL DE LA INVESTIGACIÓN Y DEL PROYECTO}

Este trabajo se enmarca en el proyecto de $\mathrm{I}+\mathrm{D}+\mathrm{i}$ del Ministerio de Economía y Competitividad Discapacidad y emprendimiento. Análisis competencial (CSO201675818-R), cuyo objetivo principal es conocer el papel que desempeñan los conocimientos, capacidades y habilidades de las personas con discapacidad física y/o sensorial en la explicación de sus acciones de emprendimiento. No obstante, esta comunicación responde a los objetivos iniciales de caracterizar a la población con discapacidad, conocer su posición en el mercado laboral y considerar el papel de las políticas de empleo en su integración sociolaboral.

Habiendo realizado una revisión de la literatura especializada sobre el tema y los datos disponibles, se pudo plantear la hipótesis de la investigación que considera que las personas con discapacidad se encuentran en una posición desfavorable a la hora de conseguir un empleo y desarrollar una actividad emprendedora. La importancia del emprendimiento como actividad económica en general y el especial significado que adquiere como vía de integración social, hace necesario abordar un análisis diferenciado.

Con el fin de llevar a cabo el análisis, se ha fijado la perspectiva competencial como factor para promover el emprendimiento de las personas con discapacidad. Ello permitirá conocer qué competencias promueven o limitan la actividad emprendedora en el colectivo y, también, analizar qué competencias y capacidades se autoatribuyen estas personas y su relación con las que se consideran relevantes para la actividad empresarial. 
A nivel metodológico, la investigación tiene un doble enfoque, cuantitativo y cualitativo. Para la fase cualitativa, se han utilizado dos técnicas principales, entrevistas en profundidad y la Técnica del Grupo Nominal (Olaz, 2007). Posteriormente, en la fase cuantitativa se ha empleado un cuestionario dirigido a personas con discapacidad.

\section{LA DISCAPACIDAD COMO CATEGORÍA DE ANÁLISIS}

Abordar el término discapacidad implica distinguir entre un conjunto de términos que, no por estar más o menos relacionados, generan los mismos campos de significatividad. Atendiendo a la Clasificación Internacional de Deficiencias, Discapacidades y Minusvalías (CIDDM) se aprecian diferentes conceptos, como son enfermedad, deficiencia, discapacidad o minusvalía. En esta clasificación, la discapacidad es entendida como el modo en que se objetiva la deficiencia en la persona, con la consiguiente repercusión directa en su capacidad para la realización de actividades consideradas normales para cualquier individuo de sus características por razón de edad, género, etc.

Según la Clasificación Internacional del Funcionamiento, de la Discapacidad y de la Salud, la discapacidad puede entenderse en la integración del modelo médico y social que la definen "como el resultado de una compleja relación entre la condición de salud de una persona y sus factores personales, y los factores externos que representan las circunstancias en las que vive esa persona" (OMS, 2001, p. 18).

Según el R. D. Legislativo 1/2013, de 29 de noviembre, por el que se aprueba el Texto Refundido de la Ley General de derechos de las personas con discapacidad y de su inclusión social, la discapacidad es una situación que resulta de la interacción entre las personas con deficiencias previsiblemente permanentes y cualquier tipo de barreras que limiten o impidan su participación plena y efectiva en la sociedad, en igualdad de condiciones con las demás.

La discapacidad puede entenderse como toda aquella limitación severa que impida realizar las actividades de la vida cotidiana y que haya durado o se prevea que se vaya a extender más de un año y tenga su origen en una deficiencia.

Desde otro punto de vista, se entiende por personas con discapacidad aquellas que poseen un grado de ésta superior o igual al 33\%, según el procedimiento de valoración establecido por R. D. 1971/1999 modificado por R. D. 1364/2012. Este Real Decreto clasifica la discapacidad en tres grandes grupos como son las físicas, las sensoriales y las psíquicas. 


\section{BREVE APROXIMACIÓN SOCIODEMOGRÁFICA AL COLECTIVO DE PERSONAS CON DISCAPACIDAD}

Según datos del INE (2016) en su estudio El Empleo de las Personas con Discapacidad basado en la explotación integrada de la Encuesta de Población Activa y de la Base Estatal de Personas con Discapacidad, en el año 2015 el número de personas en edad de trabajar (de 16 a 64 años) en España era de 30.174.500, de las que 1.774 .800 personas poseían el certificado de discapacidad, lo que representa el 5,8\% de la población total en edad laboral.

La caracterización de la población con discapacidad en edad de trabajar según género indica que existe un sesgo masculino (que no se da entre la población sin discapacidad), pues los hombres representan el 57,7\% frente al 42,3\% restante de mujeres.

En lo referente a la distribución de personas con discapacidad por tramos de edad, el colectivo más numeroso se concentra en el intervalo de 45 a 64 años donde alcanza el 69,9\%, seguido del tramo de 25 a 44 años con un $25,7 \%$, lo que parece reflejar el impacto de las discapacidades sobrevenidas. Estos datos varían si se comparan con el colectivo de personas sin discapacidad con diferencias menos acusadas entre los distintos tramos de edad.

Finalmente, resta destacar otra diferencia notable en la población de personas con discapacidad respecto a las personas sin discapacidad y que resulta de especial interés para esta investigación y es, en general, un menor nivel educativo que se manifiesta en una mayor proporción de personas con discapacidad que poseen estudios primarios, $23,9 \%$, frente al 9,6\% entre las personas sin discapacidad, así como en una menor proporción de personas con discapacidad que alcanzan estudios superiores, $15,1 \%$ frente al 32,1\% del resto de población sin discapacidad (tabla 1).

Por tanto, se está ante un colectivo que, con relación a la población sin discapacidad, se caracteriza por una mayor presencia masculina, de edad media y avanzada y que posee un nivel de estudios inferior. 
Tabla 1. Población en edad de trabajar (de 16 a 64 años) según presencia de discapacidad. 2015

\begin{tabular}{|l|c|c|c|c|c|c|}
\hline \multirow{2}{*}{} & \multicolumn{2}{|c|}{ Total } & \multicolumn{2}{c|}{$\begin{array}{c}\text { Personas sin } \\
\text { discapacidad }\end{array}$} & \multicolumn{2}{c|}{$\begin{array}{c}\text { Personas con } \\
\text { discapacidad }\end{array}$} \\
\hline Sexo & No & $\%$ & № & $\%$ & № $^{\text {o }}$ & $\%$ \\
\hline Mujeres & 15.047 .100 & 49,9 & 14.296 .100 & 50,3 & 751.000 & 42,3 \\
\hline Hombres & 15.127 .400 & 50,1 & 14.103 .600 & 49,7 & 1.023 .800 & 57,7 \\
\hline Total & 30.174 .500 & 100 & 28.399 .700 & 100 & 1.774 .800 & 100 \\
\hline Edad & No & $\%$ & No & $\%$ & № & $\%$ \\
\hline $16-24$ & 4.007 .700 & 13,3 & 3.923 .200 & 13,8 & 84.500 & 4,8 \\
\hline $25-44$ & 13.535 .700 & 44,9 & 13.079 .800 & 46,1 & 455.900 & 25,7 \\
\hline $45-64$ & 12.631 .100 & 41,9 & 11.396 .700 & 40,1 & 1.234 .400 & 69,6 \\
\hline Total & 30.174 .500 & 100 & 28.399 .700 & 100 & 1.774 .800 & 100 \\
\hline N. Estudios & № & $\%$ & № & $\%$ & № & $\%$ \\
\hline Analfabetos & 244.100 & 0,8 & 141.700 & 0,5 & 102.400 & 5,8 \\
\hline Primarios & 3.161 .600 & 10,5 & 2.736 .800 & 9,6 & 424.800 & 23,9 \\
\hline Secundaria & 17.076 .400 & 56,6 & 16.096 .400 & 56,7 & 980.000 & 55,2 \\
\hline Superiores & 9.692 .400 & 32,1 & 9.424 .700 & 33,2 & 267.700 & 15,1 \\
\hline Total & 30.174 .500 & 100 & 28.399 .700 & 100 & 1.774 .800 & 100 \\
\hline
\end{tabular}

Fuente: Elaboración propia a partir de la operación estadística "El empleo de las personas con discapacidad" (INE, 2016)

Una variable específica de notable importancia a considerar del colectivo es el grado de discapacidad de aquellos que se encuentran en edad laboral (tabla 2). La mayor parte, un 32,9\% tendría reconocido un grado de discapacidad de entre el $33,0 \%$ y el $44,0 \%$, lo que se traduce en un volumen de 583.100 personas. No obstante, cabe destacar un significativo $21,7 \%$ sobre el que no consta el grado de discapacidad. 
EL EMPRENDIMIENTO EN PERSONAS CON DISCAPACIDAD. UNA APROXIMACIÓN AL CONTEXTO SOCIOLABORAL

Tabla 2. Población en edad de trabajar (de 16 a 64 años) según grado de discapacidad. 2015

\begin{tabular}{|l|c|c|}
\hline \multicolumn{1}{|c|}{ Grado discapacidad } & № & $\%$ \\
\hline De 33\% a 44\% & 583.100 & 32,9 \\
\hline De 45\% a 64\% & 252.100 & 14,2 \\
\hline De 65\% a 74\% & 369.200 & 20,8 \\
\hline 75\% y más & 184.900 & 10,4 \\
\hline No consta & 385.400 & 21,7 \\
\hline Total & 1.774 .800 & 100 \\
\hline
\end{tabular}

Fuente: Elaboración propia a partir de la operación estadística "El empleo de las personas con discapacidad" (INE)

En relación con el mercado de trabajo, hay una gran diferencia en el colectivo de personas con discapacidad y es su menor actividad respecto a las personas sin discapacidad (Tabla 3). De este modo, solo un tercio del colectivo es laboralmente activo, $33,9 \%$ frente al $78,1 \%$ de las personas sin discapacidad, lo que representa un elemento clave a considerar en cualquier medida de promoción de su integración sociolaboral.

Tabla 3. Población en edad de trabajar (de 16 a 64 años) en relación con la actividad. 2015

\begin{tabular}{|l|c|c|c|c|c|c|}
\hline & \multicolumn{2}{|c|}{ Total } & \multicolumn{2}{c|}{$\begin{array}{c}\text { Personas sin } \\
\text { discapacidad }\end{array}$} & \multicolumn{2}{c|}{$\begin{array}{c}\text { Personas con } \\
\text { discapacidad }\end{array}$} \\
\hline & № & $\%$ & № & $\%$ & № & $\%$ \\
\hline Activos & 22.767 .100 & 75,5 & 22.166 .000 & 78,1 & 601.100 & 33,9 \\
\hline Inactivos & 7.407 .300 & 24,5 & 6.233 .700 & 21,9 & 1.173 .600 & 66,1 \\
\hline Total & 30.174 .500 & 100 & 28.399 .700 & 100 & 1.774 .800 & 100 \\
\hline
\end{tabular}

Fuente: Elaboración propia a partir de la operación estadística "El empleo de las personas con discapacidad" (INE) 


\section{LA SITUACIÓN SOCIOLABORAL DE LAS PERSONAS CON DISCAPACIDAD}

La discapacidad es un fenómeno multidimensional que afecta a la persona desde una perspectiva biológica, psicológica y social. De todas ellas, la dimensión social ha sido una de las menos estudiadas (Vázquez, 2008). Los estudios sociológicos sobre el tema están poco desarrollados, de manera que, frecuentemente, se hace referencia a la necesidad de dar visibilidad a un "sujeto ausente" (Morris, 1992). Sin embargo, la determinación de los factores sociales sobre los biológicos y psicológicos es clara. De ahí la oportunidad de estudios que propicien actuaciones encaminadas a la normalización e integración plena de las personas con discapacidad en los entornos en los que interactúan. El entorno laboral resulta clave para conseguir dicha normalización.

A continuación, se analiza la situación de las personas con discapacidad en relación con variables laborales básicas y, en general, las cuestiones relacionadas con las condiciones de trabajo.

Para ello, se ha utilizado la información que contienen las encuestas de empleo de las personas con discapacidad elaboradas por el Instituto Nacional de Estadística (INE) en el período comprendido entre 2008 y $2015^{1}$.

El porcentaje de personas con certificado de discapacidad en edad de trabajar en 2015 en España se situó en el 5,8\% de la población total en edad laboral, pero la población laboralmente activa solo supone algo más de un tercio $(33,9 \%)$, o lo que es lo mismo, menos de la mitad que las personas sin discapacidad $(78,1 \%)$. La evolución de la tasa de actividad de las personas con discapacidad desde 2008 ha seguido un ritmo ligeramente ascendente hasta 2013, año que marca un punto de inflexión en esta tendencia. En 2014 se produjo un descenso de 3,8 puntos porcentuales, lo que no se observa en la tasa de actividad de las personas sin discapacidad, que ha mantenido su crecimiento a pesar de la crisis iniciada en 2007.

En cuanto a la ocupación del colectivo, la tasa de empleo de las personas con discapacidad en España en 2015 fue del 23,4\%. Ello supone aproximadamente un tercio de la tasa de ocupación de las personas sin discapacidad. Al igual que entre estas últimas, la evolución del empleo ha sido negativa desde 2008, coincidiendo con el largo período de crisis económica. En dicho intervalo, ambos colectivos han perdido un porcentaje similar de ocupación, algo más entre las personas sin discapacidad (5,5 entre las personas sin discapacidad y 4,6 entre

\footnotetext{
${ }^{1}$ En la serie temporal de las estadísticas de "El empleo de las personas con discapacidad" existen dos rupturas metodológicas: 2008, 2009-2014 y 2015. No obstante, los datos del año 2014 han sido recalculados, según INE, utilizando la metodología 2015.
} 
los discapacitados). En 2015 se observó una ligera mejora en el porcentaje de ocupados en ambos casos.

En cuanto a la situación profesional es, mayoritariamente, de asalariados. Sobre el total de las personas con discapacidad ocupadas, el empleo por cuenta propia apenas supone algo más del $11 \%$ en todo el período considerado.

El empresario con asalariados supone en torno al 25\% de media en el periodo analizado. La evolución del empleo autónomo sin asalariados ha sido regularmente ascendente desde 2008, salvo el ligero bache producido en 2012 y 2014. Sin embargo, no ha sido esta la tendencia del empleo por cuenta propia con asalariados, de evolución irregular y con un descenso en el último año considerado (2015) por debajo del nivel de 2008. Estos datos identifican un tipo de emprendimiento bastante débil (próximo al autoempleo) con escasa capacidad de creación de empleo asalariado.

En cuanto al tipo de actividad desempeñada, está muy orientada al sector servicios. El 80,7\% de las personas con discapacidad trabajaba en actividades relacionadas con este sector en 2015. La ocupación en la industria es algo superior a un $12 \%$ en ese mismo año y ha perdido efectivos desde 2008. Por su parte, construcción y agricultura (con una ocupación del 3,8\% y 2,8\% respectivamente en 2015) son sectores de carácter residual para este colectivo.

En relación con la actividad económica desarrollada por las personas con discapacidad, más del 40\% de estas personas están ocupadas en actividades que requieren baja cualificación y/o responsabilidad (tabla 4). Actividades tales como la restauración, servicios personales, protección y venta ocupan en 2015 al 22,2\% de las personas con discapacidad. Junto a éstas, las ocupaciones elementales $(20,7 \%)$ son las actividades que centran el empleo del colectivo.

En resumen, respecto a la actividad laboral, se observa la menor tasa de ocupación del colectivo de personas con discapacidad respecto a las que no la tienen, pues supone un tercio, aproximadamente. Atendiendo a la situación profesional, las personas con discapacidad son mayoritariamente asalariadas y solo el $11 \%$ trabaja por cuenta propia y, por sectores, los servicios acaparan el mayor porcentaje del empleo en este colectivo, especialmente en tareas de media y baja cualificación.

En cuanto al desempleo, la crisis ha pasado una costosa factura al colectivo de personas con discapacidad. Su nivel de paro se ha duplicado en el período de crisis económica, pasando de un 16,3\% en 2008 al 31,0\% en 2015. Según los datos del INE para este último año, esta tasa supera diez puntos porcentuales la de las personas sin discapacidad.

Finalmente, también caben destacar las altas tasas de inactividad de las personas con discapacidad, lo que es indicativo de las dificultades que tienen para su inserción en el mercado de trabajo. 
Tabla 4. Población ocupada con discapacidad por ocupación CNO-11 (2011-2015). En porcentajes

\begin{tabular}{|l|c|c|c|c|c|}
\hline & 2011 & 2012 & 2013 & 2014 & 2015 \\
\hline Directores y gerentes & 3,1 & 2,7 & 2,3 & 2,2 & 2,3 \\
\hline $\begin{array}{l}\text { Técnicos y profesionales científicos e inte- } \\
\text { lectuales }\end{array}$ & 12,4 & 13,2 & 12,9 & 11,0 & 12,0 \\
\hline Técnicos, profesionales de apoyo & 8,6 & 8,2 & 7,3 & 7,1 & 7,0 \\
\hline $\begin{array}{l}\text { Empleados contables, administrativos y otros } \\
\text { empleados de oficina }\end{array}$ & 17,5 & 15,6 & 17,1 & 18,3 & 17,0 \\
\hline $\begin{array}{l}\text { Trabajadores de los servicios de restauración, } \\
\text { personales, protección y vendedores }\end{array}$ & 19,3 & 19,8 & 20,7 & 22,2 & 22,2 \\
\hline $\begin{array}{l}\text { Trabajadores cualificados en el sector agríco- } \\
\text { la, ganadero, forestal y pesquero }\end{array}$ & 1,5 & 3,1 & 3,1 & 2,9 & 2,6 \\
\hline $\begin{array}{l}\text { Artesanos y trabajadores cualificados de las } \\
\text { industrias manufactureras y la construcción } \\
\text { (excepto operadores de instalaciones y ma- } \\
\text { quinaria) }\end{array}$ & 8,2 & 9,0 & 10,0 & 8,7 & 8,7 \\
\hline $\begin{array}{l}\text { Operadores de instalaciones y maquinaria, y } \\
\text { montadores }\end{array}$ & 8,2 & 8,9 & 7,9 & 7,8 & 7,6 \\
\hline Ocupaciones elementales & 100 & 100 & 100 & 100 & 100 \\
\hline Total & 19,5 & 18,8 & 19,8 & 20,7 \\
\hline
\end{tabular}

Fuente: Elaboración propia a partir de INE. El Empleo de las Personas con Discapacidad

\section{CONDICIONES CONTRACTUALES}

La condición de asalariado es la más común entre las personas con discapacidad (también entre los no discapacitados) si se observa la evolución de contrataciones a personas con discapacidad entre 2008 y 2015. Resulta significativa la diferencia por sexo en dicha evolución, ya que mientras la contratación de hombres ha seguido una evolución positiva -aun en los momentos más críticos de la crisis-, la contratación de mujeres con discapacidad ha ido cayendo desde 2008 hasta suponer un 22\% menos que la de hombres en 2015.

La contratación temporal es una forma muy frecuente de contratación en este colectivo, tanto en hombres como en mujeres. Entre los primeros, supera el 90\% de los contratos celebrados en los últimos años, con una ligera ventaja en el caso 
de las mujeres. Como en el colectivo de personas sin discapacidad, resulta una fórmula de contratación cada vez más extendida, especialmente en un momento de crisis. No obstante, ha seguido una progresión ascendente en 2014 y 2015, una vez que la situación económica comienza a mejorar.

También ha ido en aumento la contratación a tiempo parcial en paralelo al descenso en la contratación a jornada completa, que pierde más de diez puntos porcentuales entre 2008 y 2015, evidenciando la precarización en las condiciones laborales de estas personas.

Fruto del ascenso en estas formas contractuales, el índice de rotación de este colectivo ha experimentado un aumento constante, lo que indica que el número medio de contratos por persona es mayor. En 2013 este índice supera los dos puntos y continúa en ascenso hasta el 2,1 en 2015.

\section{CONDICIONES SALARIALES}

Entre los efectos de la crisis, se cuenta la pérdida de capacidad adquisitiva de la población debido a la "congelación” y/o descenso salarial. En la tabla 5 se observa que la ratio salarial entre las personas con y sin discapacidad ha ido en descenso entre 2010 y 2016 (90,0 a 82,9 respectivamente), lo que implica que los salarios de uno y otro colectivo se han ido alejando entre sí, aunque pareció que en el año 2015 iba a iniciarse una recuperación. Dicha caída ha sido mayor entre las mujeres que entre los hombres, aunque la ratio de las primeras indica una mayor proximidad salarial entre uno y otro colectivo.

\section{Tabla 5. Ratio salarial entre personas con y sin discapacidad por sexo (2010-2016)}

\begin{tabular}{|l|l|l|l|l|l|l|l|}
\hline \multicolumn{1}{|c|}{ Sexo } & 2010 & 2011 & 2012 & 2013 & 2014 & 2015 & 2016 \\
\hline Ambos sexos & 90,0 & 88,5 & 85,5 & 83,9 & 82,9 & 84,3 & 82,9 \\
\hline Hombres & 83,2 & 83,3 & 79,5 & 79,5 & 79,2 & 79,6 & 79,0 \\
\hline Mujeres & 98,0 & 93,7 & 91,5 & 87,9 & 84,5 & 88,4 & 86,0 \\
\hline
\end{tabular}

Fuente: Elaboración propia a partir de INE. El Salario de las Personas con Discapacidad

La baja intensidad laboral en las personas con discapacidad resulta significativamente mayor que en las personas sin discapacidad. El empeoramiento en las posibilidades de conseguir un empleo debido a la crisis ha hecho que esta baja intensidad haya ido en ascenso desde 2008 en ambos colectivos, pero especialmente entre las personas con discapacidad, ejemplificando con ello las dificultades con 
las que se encuentra este colectivo para conseguir un empleo y, con ello su integración social.

\section{BARRERAS AL EMPRENDIMIENTO EN PERSONAS CON DISCAPACIDAD}

El colectivo de personas con discapacidad arrastra determinadas barreras que dificultan su inclusión sociolaboral. En este sentido, Mercado et al. (2013) destacan el menor nivel educativo y una cualificación profesional más alejada de las demandas del sistema productivo; la existencia de estereotipos o prejuicios sociales relativos a su potencial laboral; las dificultades de accesibilidad; el incumplimiento de las medidas de discriminación positiva o el propio desarrollo de políticas pasivas que persuaden para el desarrollo de una alternativa laboral. A estas barreras, los autores añaden y destacan dos factores que inciden en las dificultades de inserción laboral de las personas con discapacidad, como son, en primer lugar, la dispersión de los recursos de información dirigidos al colectivo, que dificultan la obtención de una visión global de las posibilidades de integración en la actividad productiva y, en segundo término, las expectativas personales y familiares con respecto a su participación en el mercado de trabajo.

No obstante, se han realizado algunos trabajos que han tratado, de manera más específica, las barreras al emprendimiento en el colectivo de personas con discapacidad. En este sentido, Moreno y Tejada (2015) coinciden en algunas de estas barreras en lo que se refiere al fomento del emprendimiento de manera específica para las personas con discapacidad, como son:

- Falta de financiación para la idea de negocio.

- Falta de formación, no tanto sobre aspectos relativos al emprendimiento como a la capacidad de autogestión y planificación ocupacional del emprendedor con discapacidad.

- Escasa implicación de los poderes públicos en el fomento del emprendimiento como alternativa profesional para el colectivo.

Por tanto, abogan por una legislación y desarrollo de medidas de apoyo activo al autoempleo y al emprendimiento de las personas con discapacidad, si bien, no tanto como un espacio exclusivo del colectivo, sino porque las personas con discapacidad participen en igualdad de condiciones y con un enfoque inclusivo de las estructuras creadas para el fomento de la actividad emprendedora.

Existen otras barreras de carácter específico que actúan sobre el desarrollo de la actividad emprendedora en personas con discapacidad. Al menos así es recogido 
por la propia Comisión Europea y la OECD/European Union (2014), entre las que se pueden encontrar:

- Falta de confianza y la configuración de aspiraciones más limitadas por parte de las personas con discapacidad.

- Condicionamientos en la percepción de los beneficios posibles, atribuyendo un mayor "riesgo" o inseguridad a la actividad por cuenta propia y, por ende, una menor tendencia a su elección como vía de integración sociolaboral.

- Carencias formativas y competenciales vinculadas a la gestión empresarial, fruto de niveles educativos inferiores o de una carencia de experiencia profesional.

- Dificultades financieras y de recursos iniciales derivadas de la desventaja social en el nivel educativo y el acceso al mercado de trabajo, así como de las dificultades de accesibilidad a las fuentes de información.

- Posible discriminación por parte de los consumidores.

- Posible aumento de costes laborales, si requieren de más personal de apoyo para desarrollar la actividad.

- Ausencia de orientadores o asesores en materia emprendedora que puedan disuadir a las personas con discapacidad, fruto de una visión estereotipada de las mismas.

Dada la existencia de estas barreras, cabe considerar la atención prestada por los poderes públicos. En este sentido, la atención en materia de integración sociolaboral del conjunto de personas con discapacidad se puede decir que acumula en nuestro país tradición democrática, ya que el art. 49 de la Constitución Española fija la responsabilidad de los poderes públicos de realizar una política de integración del colectivo. Así mismo, ya en 1982 se aprueba la Ley de Integración Social del Minusválido (LISMI) que configura al colectivo de personas con discapacidad como objetivo de las políticas de empleo.

En la actualidad, la norma de referencia es el Real Decreto Legislativo 1/2013, de 29 de noviembre, por el que se aprueba el Texto Refundido de la Ley General de derechos de las personas con discapacidad y de su inclusión social. El art. 37 de dicho texto, relativo a los tipos de empleo de las personas con discapacidad, establece que será finalidad de la política de empleo aumentar las tasas de actividad y de ocupación e inserción laboral de las personas con discapacidad, así como mejorar la calidad del empleo y dignificar sus condiciones de trabajo, combatiendo activamente su discriminación. Entre los tipos de empleo que el texto reconoce se encuentran: 
- Empleo ordinario, en empresas o administraciones públicas, incluido los servicios de empleo con apoyo. Se hace referencia a las medidas de adaptación del puesto de trabajo y accesibilidad a la empresa; el empleo con apoyo, o lo que es lo mismo, acompañamiento individualizado en el puesto de trabajo para facilitar su adaptación, y la cuota de reserva del $2 \%$ de los puestos que oferten las empresas y organizaciones de carácter público o privado de 50 o más trabajadores.

- Empleo protegido, en centros especiales de empleo que realizan una actividad productiva de bienes o servicios, participando de forma regular en el mercado y asegurando empleo remunerado a personas con discapacidad, y en enclaves laborales, que se desarrollan a través del contrato entre una empresa del mercado ordinario y un Centro Especial de Empleo para la realización de obras o servicios, para la cual, trabajadores con discapacidad del Centro Especial de Empleo, se desplazan temporalmente al centro de trabajo de la empresa colaboradora, representando un modelo de transición del empleo protegido al empleo ordinario.

- Empleo autónomo. En este sentido, el texto recoge que los poderes públicos en el ámbito de sus respectivas competencias adoptarán políticas de fomento del trabajo autónomo de personas con discapacidad dirigidas al establecimiento y desarrollo de iniciativas económicas y profesionales por cuenta propia, o a través de entidades de la economía social.

Sin embargo, en este último caso referido al empleo autónomo, cabe destacar la ausencia de concreción de actuaciones, frente a las modalidades anteriores que se encuentran con un nivel de definición y concreción mucho más elevado.

De este modo, el sistema de integración laboral, políticamente definido, de las personas con discapacidad presenta, como rasgo propio, una doble vía de integración: la ordinaria y la protegida. Ballester et al. (2014) destacan que, a pesar de esta tríada, el empleo autónomo se incluye, a efectos sistemáticos, como ámbito de integración de empleo ordinario. Y, en este sentido, realizan una consideración de interés para este trabajo y es que, en esta doble vía se ha producido, tradicionalmente, una prevalencia en la integración de las personas con discapacidad en empresas ordinarias, y solo en caso de que ello no fuera posible, de manera subsidiaria, se prevé la integración en el mercado de trabajo protegido a través de los centros especiales de empleo. Sin embargo, consideran que con el R. D. Legislativo 1/2013 la preferencia por el empleo ordinario se diluye y parece situarse al mismo nivel que el empleo protegido.

Hay otros textos emanados de los poderes públicos, generales o específicos, que pueden relacionarse con la discapacidad y el autoempleo o emprendimiento. Así, y sin ánimo de presentar una relación exhaustiva, desde el Estatuto del Trabajo 
Autónomo se comenta que la elaboración de la política de fomento del trabajo autónomo prestará especial atención a los colectivos de personas desfavorecidas, entre los cuales las personas con discapacidad ocupan un lugar preferente. Por su parte, el R. D. Legislativo 3/2015, de 23 de octubre, por el que se aprueba el texto refundido de la Ley de Empleo, establece que la política de empleo es el conjunto de decisiones adoptadas por el Estado y las comunidades autónomas para conseguir el pleno empleo, la adecuación cuantitativa y cualitativa de la oferta y demanda de empleo, la reducción y la debida protección del desempleo. Este R. D. define que las políticas activas de empleo deben dirigirse a la inclusión laboral de las personas con discapacidad, entre otros colectivos prioritarios. Por otro lado, el Plan de Acción de la Estrategia Española sobre Discapacidad (2014-2020) tiene como uno de sus objetivos operativos en materia de empleo la promoción del emprendimiento de las personas con discapacidad.

El fomento del trabajo autónomo y del emprendimiento, es una realidad asumida por las Comunidades Autónomas, generalmente en el marco de desarrollo de las políticas activas de empleo. Siguiendo a Megino y Abril (2014) en un análisis de la regulación del trabajo autónomo y la discapacidad a nivel autonómico, en el contenido de las diferentes opciones desarrolladas por los poderes públicos autonómicos para dicha finalidad, se encuentran:

- Las medidas de empuje económico, con algún modo de subvención en el inicio de la actividad.

- Medidas dirigidas a facilitar la obtención de financiación por parte de los emprendedores, procurando asimismo mejorar las condiciones de concesión.

- Otro tipo de medidas como la promoción del conocimiento y el desarrollo del trabajo autónomo a través de proyectos elaborados por terceros; tutela de emprendedores; incentivos de carácter fiscal, etc.

En tales ocasiones, indican los autores, se pueden hallar diferencias o no por colectivos específicos, como puede ser el de las personas con discapacidad y concluyen sobre la falta de una regulación propia y específica para el colectivo de personas con discapacidad, ya que la mayor parte de la regulación autonómica se limita a determinadas referencias al mismo, que no representan un tratamiento específico. Además, advierten de la ausencia, prácticamente, de tales referencias, cuando las medidas están referidas a etapas de consolidación de la actividad emprendedora.

En definitiva, las personas con discapacidad, como colectivo de especial atención, están integradas como beneficiarias en la mayor parte de los textos que regulan las medidas del fomento del autoempleo y/o emprendimiento. No obstante, hay una carencia notable en el desarrollo de programas específicos. Además, 
existe cierta dispersión normativa en materia del fomento del autoempleo y/o emprendimiento de personas con discapacidad, ya que se hacen referencias genéricas al conjunto de personas con discapacidad en la normativa más general sobre empleo y emprendimiento y, viceversa, se integran referencias genéricas al emprendimiento en la normativa referida al colectivo en general.

\section{CONCLUSIONES}

Las personas con discapacidad es uno de los colectivos más vulnerables del mercado de trabajo, experimentado un ascenso del desempleo, así como unas condiciones contractuales más desfavorables, como son la contratación temporal y a tiempo parcial y la brecha salarial con respecto a las personas sin discapacidad, así como la mayor dificultad para incorporarse con éxito -y condiciones aceptablesal mercado de trabajo. Además, esta situación parece mostrarse con un carácter estructural o crónico, motivando que apenas se produzcan cambios a lo largo del tiempo.

Ante esta situación sociolaboral, cabe preguntarse, en primer lugar, si se ha intervenido en la realidad del mismo y, en segundo término, si esta intervención ha contemplado el autoempleo o emprendimiento como una alternativa de desarrollo personal y profesional para las personas con discapacidad que favorezca su inclusión sociolaboral.

A la primera cuestión puede responderse de forma afirmativa, ya que existe una protección legal de los derechos de las personas con discapacidad y de su inclusión social. Además, el colectivo de personas con discapacidad es considerado de especial interés como destinatario de otras políticas, como por ejemplo las políticas de empleo en general.

No obstante, el sentido de la respuesta cambia en la segunda cuestión planteada. Las políticas de fomento del autoempleo o emprendimiento tienen una menor tradición en nuestro país y ello ha provocado cierta amalgama de medidas en las que, si bien se contempla a las personas con discapacidad o el emprendimiento, adolece de un enfoque integrador de ambas vertientes, como sí se ha producido en el desarrollo de las medidas de integración laboral por cuenta ajena, bien sea a través de empleo ordinario o protegido. Existe pues, una definición y concreción institucional y social del modelo de integración sociolaboral para las personas con discapacidad por cuenta ajena, pero no se ha definido, ni desde las políticas de empleo ni desde las políticas de atención al colectivo, un modelo de integración sociolaboral a través del emprendimiento.

A esta carencia en los instrumentos políticos, cabría añadir un elemento previo y necesario para que se pudieran desarrollar verdaderas políticas de fomento 
del emprendimiento en las personas con discapacidad y es un análisis previo de las barreras de especial incidencia que el colectivo tiene para desarrollar una actividad emprendedora. Entre estas se encontrarían el menor nivel educativo en conjunto y determinadas carencias competenciales; las mayores dificultades para acumular experiencia profesional; las mayores dificultades financieras asociadas su mayor dificultad de integración laboral; la protección y seguridad dada por las políticas pasivas en las expectativas de las propias personas con discapacidad; el impacto del propio entorno familiar y social; los prejuicios sociales en general y de los consumidores en particular; la formación de los técnicos y los orientadores de empleo, entre otras de carácter trasversal y que, sin duda constituyen algunos de los elementos que cualquier política o medida de fomento del emprendimiento de las personas con discapacidad ha de considerar.

Revisadas estas cuestiones, cabe advertir de la necesidad de generar una visión integral sobre la discapacidad y el emprendimiento. No hay que olvidar, que las empresas se crean porque existen personas dispuestas a asumir un riesgo y promover un negocio. Por tanto, en última instancia, la empresa se crea porque hay personas que tienen motivaciones para ello (Campos y Sanchís, 2014). Así, la motivación, es la base a partir de la cual se puede construir el emprendimiento en las personas con discapacidad y esta motivación está muy relacionada con la perspectiva competencial que se analiza en este proyecto de investigación.

\section{BIBLIOGRAFÍA}

\section{OBRAS DOCTRINALES}

Ballester, M. A., Cordero, V. \& Tomás, A. (2014). Políticas de empleo (III): Políticas destinadas a colectivos especiales (I). En Cardona, M. B. \& Cabeza, J. (Coords.), Políticas Sociolaborales (pp. 327-374). Navarra, España: Aranzadi.

Campos, V. \& Sanchís, J. R. (2014). Políticas de fomento del autoempleo. En Cardona, M. B. \& Cabeza, J. (Coords.), Políticas Sociolaborales (pp. 423-462). Navarra, España: Aranzadi.

INE. (2016). El empleo de personas con discapacidad. Notas de Prensa. Disponible en https://www.ine.es/prensa/epd_2016.pdf

Megino, D. \& Abril, M. P. (2014). Previsiones legales sobre trabajo autónomo y discapacidad a nivel autonómico. En Barrios, G. L. (Coord.), Trabajo autónomo y discapacidad (pp. 89-128). Madrid: Fundación Ramón Areces.

Mercado, E., Aizpurúa, E. \& García, L. M. (2013). Avanzando hacia la igualdad de oportunidades en la inclusión socio-laboral de las personas con discapacidad. Cuadernos de Trabajo Social, 26(1), 95-104. 
Moreno, R. \& Tejada, A. (marzo 2015). El emprendimiento como alternativa laboral de las personas con discapacidad: importancia de la ocupación productiva sobre la calidad de vida. I Congreso Nacional sobre Empleo de las Personas con Discapacidad, organizado por Ministerio de Sanidad, Servicios Sociales e Igualdad y COCEMFE, Granada, España.

Morris, J. (1992). Personal and Political: a feminist perspective on researching physical disability. Disability, Handicap $\mathcal{E}$ Society, 7(2), 157-166.

OECD/European Union. (2014). Policy Brief on Entrepreneurship for People with Disabilities. Disponible en https://www.oecd.org/cfe/leed/Policy-brief-entrepreneurship-people-disabilities.pdf.

Olaz, A. (2007). La técnica del grupo nominal como herramienta de investigación cualitativa. Madrid: Bohodón.

OMS (2001). Clasificación Internacional del Funcionamiento, de la Discapacidad y de la Salud. Disponible en http:/www.imserso.es/InterPresent2/groups/imserso/ documents/binario/435cif.pdf.

Vázquez, M. A. (2008). La construcción social de la discapacidad: Habitus, estereotipos y exclusión social. Nómadas. Revista Crítica de Ciencias Sociales y Jurídicas, 17(1), 221-232.

\section{LEGISLACIÓN}

Ley 56/2003 de Empleo Jefatura del Estado «BOE» núm. 301, de 17 de diciembre de 2003 Referencia: BOE-A-2003-23102

Real Decreto 1971/1999, de 23 de diciembre, de procedimiento para el reconocimiento, declaración y calificación del grado de discapacidad

Real Decreto 1364/2012, de 27 de septiembre, por el que se modifica el Real Decreto 1971/1999, de 23 de diciembre, de procedimiento para el reconocimiento, declaración y calificación del grado de discapacidad.

Real Decreto Legislativo 1/2013, de 29 de noviembre, por el que se aprueba el Texto Refundido de la Ley General de derechos de las personas con discapacidad y de su inclusión social. 